\title{
Citation Recommendation without Author Supervision
}

\author{
Qi $\mathrm{He}^{\dagger}$ \\ Daniel Kifer ${ }^{\S}$ \\ Jian $\mathrm{Pe}^{\ddagger}$ \\ Prasenjit Mitra ${ }^{\dagger}$ \\ C. Lee Giles ${ }^{\dagger}$ \\ $\uparrow, \S$ The Pennsylvania State University, ${ }^{\ddagger}$ Simon Fraser University \\ †\{qhe, pmitra, giles\}@ist.psu.edu, sdan+wsdm11@cse.psu.edu, ${ }^{\ddagger}$ jpei@cs.sfu.ca
}

\begin{abstract}
Automatic recommendation of citations for a manuscript is highly valuable for scholarly activities since it can substantially improve the efficiency and quality of literature search. The prior techniques placed a considerable burden on users, who were required to provide a representative bibliography or to mark passages where citations are needed. In this paper we present a system that considerably reduces this burden: a user simply inputs a query manuscript (without a bibliography) and our system automatically finds locations where citations are needed. We show that the naïve approaches do not work well due to massive noise in the document corpus. We produce a successful approach by carefully examining the relevance between segments in a query manuscript and the representative segments extracted from a document corpus. An extensive empirical evaluation using the CiteSeerX data set shows that our approach is effective.
\end{abstract}

\section{Categories and Subject Descriptors}

H.3.3 [Information Storage and Retrieval]: Information Search and Retrieval

\section{General Terms}

Algorithms, Design, Experimentation

\section{Keywords}

Bibliometrics, Context, Extraction, Recommender Systems

\section{INTRODUCTION}

One niche in online search is a literature search of academic papers. A standard approach is to perform keyword searches to retrieve documents, manually review them, and then follow the chain of bibliographic citations. This is a labor-intensive process which is especially challenging for newcomers in a research area. What we would like is a system that can automatically recommend citations for a

Permission to make digital or hard copies of all or part of this work for personal or classroom use is granted without fee provided that copies are not made or distributed for profit or commercial advantage and that copies bear this notice and the full citation on the first page. To copy otherwise, to republish, to post on servers or to redistribute to lists, requires prior specific permission and/or a fee.

Copyright 200X ACM X-XXXXX-XX-X/XX/XX ...\$10.00. manuscript that we have written without forcing us to do most of the work. Such a citation recommendation system can substantially improve both the effectiveness and efficiency of literature search. For example, the system can bring up some literatures that the authors are not aware of.

In this paper we present a system that takes a manuscript (referred to as the query manuscript) as input and automatically identifies where citations are needed and provides a list of candidate documents to be cited. Unlike previous work $[15,26,24,31,25,9]$, we do not require that the authors of the query manuscript provide the system with a partial but representative bibliography and we also do not require them to identify locations where a citation is needed. Thus we seek to minimize the users' burden in performing a literature search.

In our prior work [9], we addressed the issue of recommending documents if we knew which parts of the query manuscript required citations. In this paper, we build upon [9] by eliminating the requirement that a user marks passages that require citations. Thus our goal is to automatically analyze a manuscript lacking a bibliography and to suggest locations where citations are needed. These locations (and the words surrounding them) can also serve as explanations for why certain citations were recommended. When combined with the retrieval techniques developed in our earlier work [9], which take these candidate citation contexts as input, we can achieve a system that automatically recommends citations for a query manuscript and also identifies where citations should go. This places the least amount of burden on a user compared to other citation recommendation techniques.

Technically, a citation context in a document $d$ is a snippet of words surrounding a reference to another document $d^{\prime}$. Such a snippet can be a very accurate description of some of the ideas and contributions of $d^{\prime}$. In a query manuscript, a candidate citation context is a passage that seems to describe an idea already present in the literature (and thus in need of citation). Thus, the essence of our approach is to generate candidate citation contexts by properly comparing a query manuscript to millions of citation contexts that can be extracted from a large corpus of documents such as CiteSeerX.

The naïve approaches do not work well. The comparisons (e.g., cosine similarity) between text in a query manuscript and citation contexts extracted from a document corpus produce unreliable results because individual citation contexts can contain a lot of noise. On the other hand, we have found that direct comparisons to abstracts and the full-text of doc- 
uments in the corpus are also unreliable [9]. Abstracts are generally vague and, due to space constraints, do not adequately describe the contributions and techniques of a document. The full-text of a document in a corpus usually contains noise in the form of additional information that is irrelevant to the citation recommendation task; this frequently includes discussions of notation, experimental setups, etc. The full-text of a document can also contain many ideas but only a few of them may be relevant to the query manuscript. Thus the full-text would need to be segmented into homogeneous regions representing these ideas. This makes retrieval based on full-text similarity very slow and error-prone. In contrast, we can think of citation contexts as the equivalent of a homogeneous segment of full-text that describes one idea.

To the best of our knowledge, this is the first paper that seeks to identify candidate locations in a query manuscript where citations are needed, which is a challenging problem. We propose several techniques of increasing refinement to identify candidate citation contexts in a query manuscript. We produce a successful approach for finding candidate citation contexts in a query manuscript by carefully evaluating the relevance between passages in the query manuscript and citation contexts extracted from a document corpus. Moreover, our method considers the relationships between passages in the query manuscript to reduce the redundancy in candidate citation contexts. We thoroughly evaluate these techniques using the CIteSeerX digital library.

The rest of this paper is organized as follows. We discuss related work in Section 2. We formalize the problem in Section 3 . We discuss methods for generating candidate citation contexts in Section 4 and report the empirical evaluation in Section 5. Section 6 concludes the paper.

\section{RELATED WORK}

The problem addressed in this paper is to recommend citations for a query manuscript while placing as little burden as possible on the user and to identify locations in the query manuscript where references are needed. In contrast to this, prior work on citation recommendation either required the user to produce a representative bibliography or to explicitly mark locations where citations are needed. In this section we review the related work on citation recommendation as well as some related work on link prediction, contextual analysis, and online advertising. Figure 1 illustrates the position of this paper in literature.

Many proposals for citation recommendation require a partial list $r^{\prime}$ of citations and attempt to recover the full bibliography $r \supset r^{\prime}$. There is an implicit requirement that $r^{\prime}$ is representative of the types of papers that need to be cited. McNee et al. [15] used a collaborative filtering approach based on various rating matrices including an authorcitation matrix, paper-citation matrix, and a co-citation matrix. Documents which are co-cited often with citations in $r^{\prime}$ are candidates for recommendation. Zhou et al. [31] propagated positive labels ( the existing citations in $r^{\prime}$ ) through multiple graphs such as the paper-paper citation graph, the author-paper bipartite graph, and the paper-venue bipartite graph, and learned the labels for documents (relevant/not relevant) with respect to a given testing document in a semisupervised manner. Torres et al. [26] used a combination of context-based and collaborative filtering algorithms that used citations to build a recommendation system, and re-

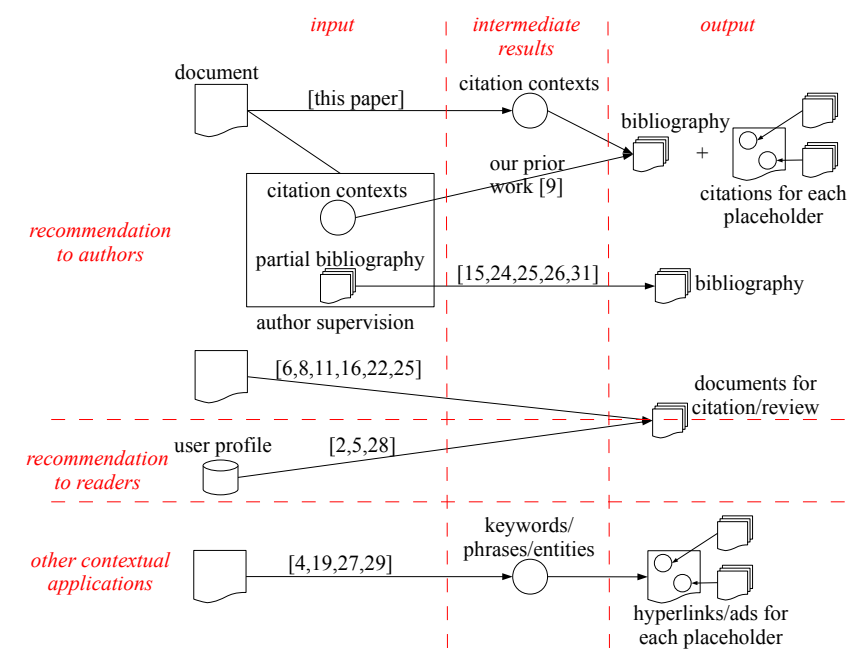

Figure 1: Position of this paper in literature.

ported that the hybrid algorithms performed better than individual ones. Strohman et al. [24] experimented with a citation recommendation system where the relevance between two documents is measured by a linear combination of text features and citation graph features. They concluded that similarity between bibliographies of documents and Katz distance [14] are the most important features. Tang and Zhang [25] explored recommending citations for specific locations in a query manuscript. However, a user must provide a representative bibliography as this information is used to compute features in the hidden layer of a Restricted Boltzmann Machine before predictions can be made.

Shaparenko and Joachims [22] proposed a technique to measure the influence of documents in a corpus on a query manuscript $d$. Their technique does not require a partial bibliography. Instead, they use language modeling and convex optimization to recommend documents. However, this technique does not identify passages where citations are needed. Scalability is also an issue with this technique and so documents are initially weeded out by textual similarity (either full-text or abstract) to the query manuscript. Full-text similarity is too slow for large digital libraries, while similarity based on abstracts yields poor recall [9] (because an abstract does not contain enough information to identify much of the related literature).

Topic models [3] provide a low-dimensional representation of documents in terms of automatically discovered and comprehensible "topics". They have been extended to incorporate citation information as well and so they can also be used to recommend citations. The aforementioned work of Tang and Zhang [25] fits in this framework. Nallapati et al. [16] introduced a model called Pairwise-Link-LDA which models the presence or absence of a link between every pair of documents and thus does not scale to large digital libraries. It also introduced a simpler model that is similar to the work of Cohn and Hofmann [6] and Erosheva et al. [8]. Here citations are modeled as a sample from a probability distribution associated with a topic. Thus, a paper can be associated with topics when it is viewed as a citation. It can also be associated with topics from the analysis of its text. However, there is no mechanism to enforce consistency 
between the topics assigned in those two ways. Kataria et al. [11] extended topic models to handle citation contexts as well and thus have improved capacity for recommending citations. However, these models cannot identify locations where citations are needed. In general, topic models require a long training process using iterative techniques such as Gibbs Sampling, EM, or variational inference and must be periodically retrained as new documents are added to the digital library.

Other studies recommend documents using user profiles rather than query manuscripts. Basu et al. [2] focused on recommending conference paper submissions to reviewers based on paper abstracts and reviewer profiles that were extracted from the Web. This is a specific step in a more general problem known as the Reviewer Assignment Problem, surveyed by Wang et al. [28]. Chandrasekaran et al. [5] presented a technique to recommend technical papers to readers whose profile information is stored in CiteSeer. Publication records are used to model a user's profile. User profiles and documents are presented as hierarchical concept trees with predefined concepts from the ACM Computing Classification System. The similarity between a user profile and a document is measured by the weighed tree edit distance.

Studies that use citation contexts to analyze papers have motivated our work. Previous studies have clearly indicated that citation contexts are a good summary of the contributions of a cited paper. Huang et al. [10] observed that citation contexts can effectively help to avoid "topic drifting" in clustering citations into topics. Aya et al. [1] noted that citation contexts can reflect the information needs (i.e., motivations) of citations and built a machine learning algorithm to automatically learn the motivation functions (e.g., compare, contrast, use of the citation, etc.) of citations by using features of citation contexts. Ritchie [21] extensively examined the impact of various citation context extraction methods on the performance of information retrieval tasks. In [9], we studied how to use citation contexts to recommend papers to cite. That work assumed that a user has provided a query manuscript (without bibliography) that indicates locations where citations are needed. Then, we designed a non-parametric probabilistic model that uses information about citation contexts to measure the relevance between two documents and also between a citation context and a document. Requiring a user to explicitly mark locations where references are needed places a burden on a user who may not be familiar with the literature and who may therefore fail to mark certain locations. Thus we seek to address this issue in the paper.

Automatically extracting citation contexts is novel approach for citation recommendation and it bears some resemblance to other domains such as hyperlink generation and content-based advertising. We only mention a few representative papers here to highlight the significant differences between these tasks and citation recommendation. For example, Brzeski et al. [27] used named entities in a document as hyperlink snippet candidates. However, in citation recommendation, the ideas that need to be cited are more complicated than named entities. Indeed, if a query manuscript contains named entities, they usually refer to established ideas in the literature that the author is already aware of; marking named entities as items that need to be cited would not provide added value to a user.

Ad placement (recommending ads for webpage) typically tries to match ad keywords to a given webpage. For example, Ribeiro-Neto et al. [19] explore techniques using external information to reduce the mismatch in vocabularies of ads and webpages. Broder et al. [4] compared each section of a Web page to the advertisements by enhancing their relevance at the taxonomy level, thus each section can be seen as an advertising context candidate. Yih et al. [29] automatically extracted keywords from a free text and associated them with advertisements. Keywords are learned using the machine learning based on a bunch of linguistic/syntactic/semantic/proximity features from text and query logs. Even though one may metaphorically view a webpage as a query manuscript and an ad as a potential paper to cite, those techniques are generally not applicable to citation recommendation. First, ads are designed to have succinct descriptions while research papers contain many different ideas that are not described as succinctly; thus the main objects involved have different characteristics. Second, the types of external datasets that are useful are also significantly different (for example query logs vs. digital libraries).

\section{PROBLEM SETUP}

In this section, we introduce notation and terminology, and describe the citation recommendation problem.

We let $d$ denote a document, which is a sequence of words. A document cites other documents $r_{1}, \ldots, r_{m}$. A citation location $\ell$ is a place in document $d$ where some document $r$ is cited as a reference. The citation context $c_{\ell}$ is the sequence of words surrounding this citation location $\ell$ and which describes the relevance of $r$ to document $d$. We say that $c_{\ell}$ is an out-link citation context with respect to $d$ because it describes an outgoing link from document $d$. Similarly, we say that $c_{\ell}$ is an in-link citation context with respect to document $r$ because it describes a link going into $r$.

Given a citation location, identifying the set of words that describes why reference $r$ is relevant to document $d$ is a challenging task and is left for future work. For the current paper, we thus use a simple but efficient heuristic: if we are given a citation location $\ell$, then the citation context $c_{\ell}$ consists of the 100 words surrounding $\ell$. Our techniques are designed to be robust to the noise introduced by this heuristic (or any other technique that delimits the citation context from the rest of the text). Using this heuristic, we can efficiently collect citation contexts from large online document repositories such as CiteSeerX.

The problem we are addressing is the following. A user submits an unlabeled query manuscript. This document contains no bibliography and no references. This document does not even contain markers indicating where references should go. Our goal is to segment the document into a sequence $<w_{1}, \ldots, w_{n}>$ of possibly overlapping windows and to label each window $w_{i}$ as either (1) a citation context or (2) not a citation context. Overlapping windows that are labeled as citation contexts are merged together. This results in a sequence of disjoint candidate citation contexts $<c_{1}^{(d)}, \ldots, c_{k_{1}}^{(d)}>$ (we use the term candidate to emphasize the fact that these are predictions). We may optionally want to assign a weight $y_{i}$ to each $c_{i}^{(d)}$ to indicate our confidence.

Once we have discovered the candidate citation contexts $c_{1}^{(d)}, \ldots, c_{k_{1}}^{(d)}$ (and confidence weights $\left.y_{1}, \ldots, y_{k_{1}}\right)$, we can use 
a large online document corpus $D$ (such as CiteSeerX) to retrieve a ranked list of relevant documents $r_{1}, r_{2}, \ldots$ The relevance $\operatorname{sim}(d, r)$ of a document $r \in D$ with in-link citation contexts $c_{1}^{(r)}, \ldots, c_{k_{2}}^{(r)}$ can be computed using the trace operator [20] relevance scoring developed in [9]:

$$
\operatorname{sim}(d, r)=\frac{1}{k_{1} k_{2}} \sum_{i=1}^{k_{1}} \sum_{j=1}^{k_{2}} y_{i}\left(c_{i}^{(d)} \cdot c_{j}^{(r)}\right)^{2}
$$

where each citation context is treated as a tf-idf word vector. If no weights $y_{i}$ are given, we can set them to 1 in Equation 1. Note that the relevance of $r$ to the query manuscript $d$ is dependent on the similarity of the discussion in $d$ to what other authors say about $r$. In practice, we add both document titles as citation contexts in Equation 1. We found that this overall approach outperforms textual similarity to document $r$ [9].

\section{FINDING CITATION CONTEXTS}

In this section we propose 4 different models for finding citation contexts $<c_{1}^{(d)}, \ldots, c_{k}^{(d)}>$ and associated confidence weights $\left\langle y_{1}, \ldots, y_{k}\right\rangle$ in an unlabeled query manuscript $d$. We start with simple baselines and progress towards more sophisticated use and generation of features culminating in our proposed approach in Section 4.4.

\subsection{Method 1: Language Models}

It is quite natural to try to use language models [17] for finding citation contexts. From CiteSeerX, we can extract 100 words around each citation that occurs in a document in this corpus. Treating these as positive examples of citation contexts, we get roughly 4 million positive training examples. In theory, we could use the remaining text in the corpus as the negative examples. However, this set of negative examples is too large for us to process and so we treat this as a one-class classification problem by building the language models only from the positive examples. Note that the positive examples are themselves noisy - not all words surrounding a citation refer to that citation (identifying which words are relevant to a citation is a difficult task to be considered in future work).

After building an $n$-gram language model $\mathrm{LM}^{1}$, we can use it to try to recognize locations in the query manuscript $d$ where citations should exist. We can do this with a two-step approach of scoring windows followed by burst-detection.

First, we divide the query manuscript into a sequence $\left\langle w_{1}, \ldots, w_{n}\right\rangle$ of overlapping windows of 100 words each. For example, $w_{1}$ would contain words 1 through 100, $w_{2}$ would contain words 2 through 101, $w_{3}$ would contain words 3 through 102, etc. Using the language model LM, we can assign a probability score $p_{i}$ to each window $w_{i}$. This gives a sequence $<p_{1}, \ldots, p_{n}>$ of scores. We now can use a burst detection method, such as the 2-state automaton model proposed by Kleinberg [13], to identify regions of relatively high probability. Each such connected region is output as a candidate citation context $c_{i}^{(d)}$ with corresponding weight $y_{i}$ given by the burst detection method [13].

\subsection{Method 2: Contextual Similarity}

\footnotetext{
${ }^{1}$ Our experiments used the Katz back-off strategy when estimating conditional probabilities [12].
}

An alternative to language models is to directly compare similarity between different pieces of text using tf-idf weighting. There are two possibilities here. The first approach is to divide a document into a sequence $\left\langle w_{1}, \ldots, w_{n}\right\rangle$ of overlapping windows (as in Section 4.1) and, for each $w_{i}$, to compute its similarity to the full text or abstract of documents in the corpus $D$. In prior work [9], we showed that this approach leads to poor retrieval of relevant documents. One of the reasons for this is that the full text or abstract of a document $r \in D$ is not necessarily the best description of the ideas in $r$. For example, the full-text of a document contains many parts that do not clearly describe or are marginally relevant to its main contribution. This can include experimental setup, discussion of notation, narratives containing specific examples, etc. On the other hand, many abstracts do not adequately describe all aspects of a paper's contribution.

In general, we have found that citation contexts that surround references to a document $r$ do a much better job of describing the important aspects of document $r$. Indeed, although a single citation context is noisy, the set of all inlink citation contexts that reference $r$ does contain quite a bit of signal. Thus a better approach is to compare a window in a query document $d$ to citation contexts extracted from an online corpus such as CiteSeerX (in fact, this was the motivation for our initial line of research [9]).

We can use the relevance model based on trace operators [9] to compute the relevance of window $w_{i}$ to the in-link citation contexts $\left\langle c_{1}^{(r)}, \ldots, c_{k_{2}}^{(r)}>\right.$ for a document $r$ in the corpus $D$ (where $w_{i}$ and the $c_{j}^{(r)}$ are treated as tf-idf vectors):

$$
\operatorname{sim}\left(w_{i}, r\right)=\frac{1}{k_{2}} \sum_{j=1}^{k_{2}}\left(w_{i} \cdot c_{j}^{(r)}\right)^{2}
$$

and we can assign a score to $w_{i}$ as either $\max _{r \in D} \operatorname{sim}\left(w_{i}, r\right)$ or $\sum_{r \in D} \operatorname{sim}\left(w_{i}, r\right) /|D|$. We have found that the latter (averaging) approach works better to assign a score for $w_{i}$.

Thus after we divide a query manuscript $d$ into overlapping windows $\left\langle w_{1}, \ldots, w_{n}\right\rangle$ (as in Section 4.1), we use $\sum_{r \in D} \operatorname{sim}\left(w_{i}, r\right) /|D|$ to assign a score $p_{i}$ for each window $w_{i}$. We again use a burst-detection method [13] on the sequence $\left\langle p_{1}, \ldots, p_{n}\right\rangle$ of scores to find regions with relatively high scores. Each such region is composed of consecutive words from $d$ and is output as a candidate citation context $c_{i}^{(d)}$. The weight $y_{i}$ assigned to context $c_{i}^{(d)}$ is the weight given to the associated burst [13].

\subsection{Method 3: Topical Relevance}

One of the problems with the contextual relevance approach from Section 4.2 is that it is very time-consuming to compare every window $w_{i}$ with all of the contexts extracted from a large document corpus. For example, in our CiteSeerX dataset, there are roughly 4 million such contexts to compare to. Thus dimensionality reduction is needed.

If we treat citation contexts as one type of object and the documents they refer to as another type of object, we get a large context-document bipartite graph. The goal is to cluster this bipartite graph to obtain clusters of citation contexts and clusters of documents. Then we must judiciously choose representatives for each cluster.

The clustering itself could be performed using, for example, a variation of topic models [3] or co-clustering. Since our 
dataset is very large, we did not find significant differences in quality and even the following approach worked well: first, cluster the citation contexts (treating each context as a bag of words), and then separately cluster the documents using their titles and abstracts.

Given citation context clusters $C_{1}, \ldots, C_{k_{1}}$ and document clusters $Z_{1}, \ldots, Z_{k_{2}}$, we can form cluster representatives as follows. For a citation context cluster $C_{i}$, its representative $\tilde{c}_{i}$ is:

$$
\tilde{c}_{i}=\frac{1}{\left|C_{i}\right|} \sum_{x \in C_{i}} x
$$

which is the average tf-idf vector representing each context in the cluster $C_{i}$. We could, in principle, form representatives $\tilde{z}_{i}$ for the document clusters $Z_{i}$ in the same way. However, this would represent a document cluster as the average of the abstracts (treated as tf-idf vectors) in the cluster. As we noted previously, citations refering to a document can do a better job than the abstract of describing a document's relevance. Thus generating document cluster representatives $\tilde{z}_{i}$ is more involved. First, we estimate conditional probabilities between clusters:

$$
p\left(C_{j} \mid Z_{i}\right)=\frac{\# \text { contexts in } C_{j} \text { citing documents in } Z_{i}}{\text { total \# contexts citing documents in } Z_{i}} ;
$$

and then we compute the representative $\tilde{z}_{i}$ for cluster $Z_{i}$ as:

$$
\tilde{z}_{i}=\sum_{j=1}^{k_{1}} p\left(C_{j} \mid Z_{i}\right) \tilde{c}_{j} .
$$

We thus use what other authors say about documents to form the cluster representatives. Now we split the query document $d$ into a sequence of overlapping windows $\left\langle w_{1}, \ldots, w_{n}\right\rangle$ and, using the probabilistic relevance model [9] based on trace operators [20], we compute a score $p_{i}$ for each $w_{i}$ as:

$$
p_{i}=\frac{1}{k_{2}} \sum_{j=1}^{k_{2}}\left(w_{i} \cdot \tilde{z}_{j}\right)^{2} .
$$

Once again we use a burst detection method [13] on the sequence $\left\langle p_{1}, \ldots, p_{n}>\right.$ of scores to find regions with relatively high scores. Each such region is composed of consecutive words from $d$ and is output as a candidate citation context $c_{i}^{(d)}$. The weight $y_{i}$ assigned to context $c_{i}^{(d)}$ is the weight given to the associated burst [13].

\subsection{Method 4: Dependency Feature Model}

Now we present our final model which is based on ideas from Sections 4.1, 4.2, and 4.3 and addresses their deficiencies. The model described in this section is based on the following two observations:

- The methods in previous sections use a single score to discriminate between contexts and non-contexts. We have found that other types of features are also correlated the presence or absence of contexts, but this correlation is nonlinear. These features are described in Section 4.4.1.

- The methods in previous sections do not relate a window $w_{i}$ with prior windows in the query manuscript. We have found that it is important to consider these relations. This is necessary to eliminate redundancy and to prevent a single theme from dominating the other themes present in a query manuscript. To see this, suppose a query manuscript frequently mentions "mixtures of multinomial distributions". Many parts of this document are therefore related to papers discussing discrete mixture models and these parts will be given high scores. All of these parts would be returned in a list of the most promising candidate citation contexts (even though this is redundant from the user's point of view). On the other hand, other parts of the query document may be related to different research areas; these parts would receive lower scores and therefore would not be returned in a list of the most promising candidate citation contexts (they would be crowded out by the "mixtures of multinomial distributions"). Thus we would like to return one candidate context representing the relationship between "mixtures of multinomial distributions" and the literature, and we would like to return other candidate citation contexts that represent different themes. It is interesting to note that actual citations also follow this pattern - a citation is often cited at the frontal references if it is mentioned multiple times in query manuscript.

Both of these issues require additional features that are combined in nonlinear ways. Some of these features represent long-range dependencies in the query manuscript. We discuss these features in Section 4.4.1, we discuss the model incorporating these dependencies in Section 4.4.2, and we turn this into an ensemble that votes using a Beta-Binomial noise model in Section 4.4.3

\subsubsection{Additional Features}

Table 1 summarizes the features we compute for every window $w_{i}$ from the sequence of overlapping windows $<$ $w_{1}, \ldots, w_{n}>$ constructed from the query manuscript $d$. The first group of features compares $w_{i}$ to the citation contexts culled from a corpus $D$ (such as CiteSeerX). Many of them use the cluster representatives $\tilde{z}_{j}$ defined in Section 4.3. These features include average similarity to all cluster representatives, to the top $K$ representatives, and statistics about n-grams that are shared between $w_{i}$ and the citation contexts from corpus $D$. The second group of features compares $w_{i}$ to the query document $d$. They range from simple cosine similarity between $w_{i}$ and $d$ to dependence on prior predictions such as maximum similarity between $w_{i}$ and a previous (candidate) citation context in $d$.

\subsubsection{Inference over long-range dependencies}

For each window $w_{i}$, let $\ell_{i}$ be its binary label (1 for citation context and 0 for non-context). For query manuscript $d$, document corpus $D$, and parameter set $\Theta$ (to be specified later), the learning model would be:

$$
\begin{aligned}
\Theta & =\operatorname{argmax}_{\Theta} p\left(\ell_{1}, \ldots, \ell_{n} \mid d, D, \Theta\right) \\
& =\operatorname{argmax}_{\Theta} \sum_{i} \log p\left(\ell_{i} \mid\left\{w_{j}: \ell_{j}=1 \wedge j<i\right\}, d, D, \Theta\right)
\end{aligned}
$$

since the label $\ell_{i}$ of window $i$ depends on the previously predicated citation contexts because of features 14, 15, 16, and 17. Since the length of the history is not bounded (i.e. the model is not Markovian), exact inference is intractable. There are two options: to approximate the model (by adding a Markovian structure) or to approximate the inference. We note that the long-range history is important - if a passage towards the end of the query manuscript is similar to a passage towards the beginning, we should only return the 


\begin{tabular}{|c|c|c|}
\hline Type & Feature of window $w_{i}$ & Description \\
\hline $\begin{array}{l}\text { Context } \\
\text { features }\end{array}$ & $\begin{array}{l}\text { 1. sumsim2topic } \\
\text { 2. sumtopksim2topic } \\
\text { 3. maxsim2topic } \\
\text { 4. avgnumtopic } \\
\text { 5. avgtopicentropy } \\
\text { 6. mintopicentropy } \\
\text { 7. maxtopicentropy } \\
\text { 8. numterm } \\
\text { 9. avgglobalfreq }\end{array}$ & $\begin{array}{l}\sum_{j=1}^{k_{2}}\left(w_{i} \cdot \tilde{z}_{j}\right)^{2} \text { (using cluster representatives } \tilde{z}_{j} \text { as defined in Section } 4.3 \text { ). } \\
\left(w_{i} \cdot \tilde{z}_{j_{1}}\right)^{2}+\cdots+\left(w_{i} \cdot \tilde{z}_{j_{K}}\right)^{2} \text { for the top } K \text { cluster representatives most similar to } w_{i} \text {. } \\
\max _{j}\left(w_{i} \cdot \tilde{z}_{j}\right)^{2} \\
\text { Avg (over } n \text {-grams common to } w_{i} \text { and contexts in } D \text { ) \# of } \tilde{z}_{j} \text { containing the } n \text {-gram. } \\
\text { Avg (over } n \text {-grams common to } w_{i} \text { and contexts in } D \text { ) of the entropy of tf-idf scores assigned by } \\
\text { the } z_{j} \text { to the } n \text {-gram. } \\
\text { Similar to avgtopicentropy (Feature } 5 \text { ) but using min instead of avg. } \\
\text { Similar to avgtopicentropy (Feature } 5 \text { ) but using max instead of avg. } \\
\# \text { of distinct } n \text {-grams common to } w_{i} \text { and contexts from } D \text {. } \\
\text { Avg (over } n \text {-grams common to } w_{i} \text { and contexts in } D \text { ) \# of contexts from } D \text { containing the } n \text {-gram. }\end{array}$ \\
\hline $\begin{array}{l}\text { Long-range } \\
\text { dependen- } \\
\text { cies with } \\
\text { query } \\
\text { manuscript }\end{array}$ & $\begin{array}{l}\text { 10. numfirstterm } \\
\text { 11. sim2doc } \\
\text { 12. avglocalfreq } \\
\text { 13. avetfidf } \\
\text { 14. depnumfirstterm } \\
\text { 15. maxsim2context } \\
\text { 16. avgsim2context } \\
\text { 17. isfirsttopic }\end{array}$ & $\begin{array}{l}\text { \# of n-grams in } w_{i} \text { that have not appeared in prior windows } w_{j}(j<i) \text { from query manuscript } d \text {. } \\
\text { Cosine similarity of } w_{i} \text { to abstract and title of } d \text {. } \\
\text { Avg (over } n \text {-grams in } w_{i} \text { ) frequency of the } n \text {-gram in query manuscript } d \text {. } \\
\left.\left.\text { Avg (over } n \text {-grams common to } w_{i} \text { and contexts from } D\right) \text { TF-IDF score (TF: over } d \text {; IDF: over } D\right) \text {. } \\
\# \text { of n-grams in } w_{i} \text { that have not appeared in prior (candidate) contexts from query manuscript } d \text {. } \\
\text { Max cosine similarity of } w_{i} \text { to prior (candidate) citation contexts in } d \text {. } \\
\text { Avg (over prior candidate contexts) cosine similarity of } w_{i} \text { to all prior (candidate) contexts in } d \text {. } \\
\text { Is } \operatorname{argmax}_{j}\left(w_{i} \cdot z_{j}\right)^{2} \text { equal to argmax }{ }_{j}\left(\hat{w} \cdot z_{j}\right)^{2} \text { for any prior (candidate) context in } d \text { ? }\end{array}$ \\
\hline
\end{tabular}

Table 1: Features for window $w_{i}$ from query manuscript $d$ computed with corpus $D$.

latter passage as a candidate citation context (to avoid redundancy); in fact, in actual documents the earlier passage is more likely to contain a reference than the passage that follows later (thus actual citation patterns are aligned with our goal of reducing redundancy in the candidate citation contexts.). Thus a Markovian assumption would not be justified and we choose to approximate the inference instead.

In order to combine features in a nonlinear way, we use an ensemble of decision trees as our base model in Equation 2 (ensemble creation is discussed in Section 4.4.3). Since actual citation patterns are aligned with our goal (reducing redundancy in the output), model training is very easy: we take a subset of documents from the corpus $D$, divide each $d \in D$ into a sequence of windows $<w_{1}, \ldots, w_{n_{d}}>$ and use the true citation information to assign a label to each window. Thus in training mode, all of the features can be computed and the learning algorithm for each tree in the ensemble is unchanged.

Inference, however, can be problematic because in Equation 2 the label of a window $w_{i}$ could depend on windows $w_{j}$ that are arbitrarily far away. We have found that the following one-pass greedy approach is efficient, simple, and works reasonably well: we start predicting from left to right, and once a window $w_{i}$ is labeled, we do not revisit (change) this label later; a window $w_{i}$ is labeled as a candidate citation context if its score (given by the ensemble) exceeds a threshold $\tau$ that is set by cross-validation.

\subsubsection{Ensemble Voting}

Since decision trees do not have algorithmical stability [18], we use an ensemble of $n$ decision trees. The individual trees are constructed

- using all of the features from Table 1;

- using only the small set of best features;

- using all but one feature;

- using bootstrapping.

For a window $w$, each tree $T_{i}$ returns a prediction saying that leaf making the prediction has $m_{i}$ out of $k_{i}$ training points labeled as citation contexts. We aggregate these scores using the following approach. There is a $p$ corresponding to the probability that $w$ is a citation context. The $m_{i}$, $k_{i}$ values for each tree are generated from a $\operatorname{Binomial}\left(p_{i}, k_{i}\right)$ distribution, where $p_{i}$ is a noisy version of $p$ and $m_{i}$ is the number of heads that occur in $k_{i}$ coin tosses.

The noisy $p_{i}$ comes from a $\operatorname{Beta}(\gamma p, \gamma(1-p))$ distribution with mean $p$ and variance $p(1-p) /(\gamma+1)$; we can think of $\gamma$ as a tuning parameter that affects the variation of the unobserved $p_{i}$ values. Under this noise model, $m_{i} / k_{i}$ is an unbiased estimator of $p$ (however the variance of the estimator depends on $p$ and $\gamma$ ). A convex combination of the $m_{i} / k_{i}$ is also an unbiased estimator of $p$, and so we would like the convex combination with minimum variance. Surprisingly, even though the variance of the estimator depends on the unknown quantity $p$, we do not need to know $p$ to form the estimator. The combined probability estimate is shown in the following lemma, whose proof is in Appendix A.

LEMMA 4.1. The minimum variance unbiased estimator of $p$ formed as a convex combination of the $m_{i} / k_{i}$ is:

$$
\frac{1}{\sum_{j=1}^{n} \frac{k_{j}}{k_{j}+\gamma}} \sum_{i=1}^{n} \frac{m_{i}}{k_{i}+\gamma} .
$$

\section{EXPERIMENTS}

\subsection{Experimental Setup}

We implemented the citation recommendation system in the CiteSeerX to evaluate its performance. All research papers that were published and crawled before year 2008 were used as the training document corpus $D$. After removing duplicates and papers for which there were no citation contexts, titles, or abstracts, we obtained 731,535 unique citing documents and 528,647 unique cited documents in the corpus. For each paper, we extracted its title and abstract as the text content. Within each paper in the corpus, the 50 words before and 50 words after each citation reference were treated as the corresponding citation context (a discussion on the number of words to use can be found in [21]). We also removed stop words from the contexts. In order to preserve the time-sensitive past/present/future tenses of verbs and the singular/plural styles of named entities, no stemming was done, but all words were transferred to lower-case. We obtained 4,175,615 unique citation contexts and 869,427 unique word terms (unigrams). Bigrams and trigrams were computed using only the top 65,535 unigrams because of 
sparsity $^{2}$. This resulted in 597, 258 bigrams and 188, 154 trigrams. The combination of unigrams and bigrams is used as the term corpus by default.

We used one set of 100 papers for decision tree construction and parameter tuning. We used another set of 100 papers from early 2008 for testing the quality of candidate citation contexts output by each method under evaluation.

We implemented all algorithms in $\mathrm{C}++$ and ran them on a PSU Linux cluster with 8 nodes, each of which has $8 \mathrm{CPU}$ processors of $2.67 \mathrm{GHz}$ and $128 \mathrm{G}$ main memory.

\subsection{Methods Being Compared}

In order to fairly compare different methods for generating candidate citation contexts, we define the concept of context coverage. The context coverage is the fraction of the words in a document $d$ that are inside a candidate citation context. Comparisons between different methods can only be fair if all of the methods generate candidate citation contexts with similar context coverage. The first 3 methods we compare are simple baselines.

- Ground truth: This method outputs the 100 words surrounding the actual citation locations. Since we evaluate all methods on how well their candidate citation contexts can be used to retrieve relevant documents, this baseline represents an upper bound on the quality that can be achieved. The context coverage of ground truth contexts over the full-text of a testing paper is $34.5 \%^{3}$. All ground truth contexts $c_{i}$ are assigned the same weight $y_{i}=1.0 \mathrm{in}$ Equation 1 to rank relevant documents from corpus $D$.

- Random: Candidate citation contexts are generated randomly until they achieve a coverage of 0.345 . Results are averaged over 10 runs. We use the weight $y_{i}=1$ for each candidate citation context $c_{i}$ in Equation 1.

- Fulltext: There is only one candidate citation context but it consists of the whole text of a testing paper; the context coverage is 1.0 .

The previous 3 baselines are used as sanity checks when evaluating the performance of the models presented in this paper. We also compare the following methods, which we tune to get a context coverage close to 0.345 .

- Language Models (LM): We build three language models as described in Section 4.1. The first language model is based on unigrams, the second is based on bigrams, and the third is based on trigrams.

- Contextual Similarity: Here we use the method described in Section 4.2 that compares a window $w_{i}$ to the citation contexts extracted from the corpus $D$ (CiteSeerX).

- Topical Relevance: This is the method described in Section 4.3. Citation contexts and documents were clustered into 1,000 clusters each using top-down bisection clustering [30].

- Dependency Feature Model: This is the method described in Section 4.4. We used C4.5 [18] to build each decision tree in the ensemble. We considered the effects of leaving out one feature on predicting citation contexts. Table 2 shows the change in accuracy when the corresponding feature is removed; negative numbers mean the

\footnotetext{
${ }^{2}$ Unigrams with a document frequency $<8$ are then cut.

${ }^{3}$ On average, there are 24.19 citation contexts in a testing paper, but they contain considerable overlap; the average length of a testing paper is 4402.7 words.
}

\begin{tabular}{|l|r|}
\hline Feature removed & $\begin{array}{r}\text { change in cross- } \\
\text { validation accuracy }\end{array}$ \\
\hline avgsim2context & $\mathbf{- 1 4 . 6 8 \%}$ \\
numfirstterm & $-8.58 \%$ \\
maxsim2topic & $-2.44 \%$ \\
depnumfirstterm & $-2.44 \%$ \\
sumsim2topic & $-2.02 \%$ \\
avetopicentropy & $-0.42 \%$ \\
sumtopksim2topic & $-0.42 \%$ \\
avenumtopic & $-0.41 \%$ \\
maxtopicentropy & $0.41 \%$ \\
avelocalfreq & $+1.21 \%$ \\
aveglobalfreq & $+4.92 \%$ \\
mintopicentropy & $+5.74 \%$ \\
numterm & $+7.28 \%$ \\
avetfidf & $+7.31 \%$ \\
maxsim2context & $+7.40 \%$ \\
isfirsttopic & $+9.78 \%$ \\
sim2doc & $+13.44 \%$ \\
\hline
\end{tabular}

Table 2: Evaluation of features. Cross-validation error using all features is $24.5 \%$.

accuracy dropped, positive numbers mean the accuracy increased (however, we have found that removing all features with a positive difference causes accuracy to decrease). We see that some of the most important features are the ones incorporating long-range dependencies between predicted labels (these include avgsim2context and depnumfirstterm). The topical relevance features are also considerably important as maxsim2topic and sumsim2topic are ranked among top 5 features. We used an ensemble of 23 decision trees: 1 decision tree using all features, 1 decision tree using the top 5 features from Table 2, 17 take-one-feature-out decision trees, and 4 bootstrapped decision trees. We set $\gamma$ to be 100 and $\tau$ to be 0.85 with cross-validation. $\tau$ is used to control the context coverage and application-dependent. We conducted $t$-tests with hypothesis that a close $\gamma$ value (against the best $\gamma=100$ achieved with cross-validation) does not change vote scores significantly. The $p$-values for $t$-tests are $0.247(\gamma=10), 0.965(\gamma=1,000), 0.512(\gamma=10,000)$ and $0.442(\gamma=100,000)$ respectively. That is to say, $\gamma$ has a wide setting range (the vote scores do not deviate much from the best ones) and is easy to be tuned in practice.

\subsection{Evaluation of Candidate Citation Context Locations}

In this section, we evaluate how well the candidate citation contexts (generated by different techniques) correspond to the actual locations where citation are needed. In Section 5.4 we will then evaluate how the quality of recommended citations that are computed based on the candidate citation contexts. To perform the evaluation, we identify the true citation locations in each of our testing documents and use the 100 words surrounding each location as the ground truth.

\subsubsection{Metrics}

Let $W_{g}$ be the part of a testing paper covered by the ground truth contexts and $W_{t}$ be the part covered by the candidate citation contexts generated by one of the methods discussed earlier. Then $W_{t} \cap W_{g}$ measures the correctness 


\begin{tabular}{|l|l|l|l|}
\hline method & CP & CR & PN ratio \\
\hline Ground Truth & 1 & 1 & $+\infty$ \\
Fulltext (coverage: 1) & 0.345 & 1 & $1 / 1.9$ \\
\hline Dependency Model & $\mathbf{0 . 4 7 2}$ & $\mathbf{0 . 5 6 1}$ & $\mathbf{1 / 1 . 1 2}$ \\
Topical Relevance & 0.427 & 0.46 & $1 / 1.34$ \\
Contextual Similarity & 0.34 & 0.355 & $1 / 1.94$ \\
Random & 0.334 & 0.356 & $1 / 2$ \\
Trigram LM & 0.273 & 0.268 & $1 / 2.66$ \\
Bigram LM & 0.272 & 0.268 & $1 / 2.68$ \\
Unigram LM & 0.221 & 0.208 & $1 / 3.52$ \\
\hline
\end{tabular}

Table 3: Evaluation of citation locations under the default context coverage of 0.345 .

of $W_{t}$ while $W_{t} \backslash W_{g}$ measures incorrect predictions. We use these quantities to define the following 3 metrics:

- Coverage Precision (CP): $\frac{\left|W_{t} \cap W_{g}\right|}{\left|W_{t}\right|}$,

- Coverage Recall (CR): $\frac{\left|W_{t} \cap W_{g}\right|}{\left|W_{g}\right|}$,

- Positive vs. Negative ratio (PN ratio): $\frac{\left|W_{g} \cap W_{t}\right|}{\left|W_{t} \backslash W_{g}\right|}$.

As discussed in Section 5.2, we try to keep the context coverage constant in order to make apples-to-apples comparisons between different methods. That is, the part $W_{t}$ of a document that is covered by the candidate citation contexts is constrained to be roughly 0.345 of the total document; this is the same fraction in the documents (in our test set) which is covered by the ground truth $W_{g}$. For this reason, coverage precision and coverage recall are not necessarily in conflict (in contrast to ordinary precision and recall measures used for other information retrieval tasks).

\subsubsection{Result Analyses}

Table 3 compares baselines and the different methods for generating candidate citation contexts. We see that the language models do not perform well for this task; in fact, they are outperformed by a random selection of citation contexts. It is not surprising that language models did not perform well - they were only trained on positive examples (citation contexts) but not on negative examples (because it was infeasible) and they did not have long-range dependencies built in. However, it was still surprising that they performed worse than random guessing.

While the unigram language model performed the worst, the bigram and trigram models had very similar performance. This provides evidence for the notion that pairs of words are commonly used to refer to established ideas (e.g., "mixture models", "information retrieval", etc.) while trigrams (e.g., "support vector machines") are not nearly as common (in a relative sense).

We see that contextual similarity (Section 4.2) has similar performance to random guessing. This implies that citation contexts are very noisy and that a dimensionality reduction approach (the methods in Section 4.3 and 4.4) would be useful for extracting signal from the noise. This intuition is also confirmed by Table 3, where the topical relevance method (Section 4.3) and the dependency feature model (Section 4.4) are the only methods which significantly outperform random guessing.

We can also see that the dependency feature model outperforms topical relevance as well, and this is due to the presence of long-range dependency features that eliminate redundancy (as discussed in Section 4.4).

The unigram, bigram, and trigram models are extremely fast, as is random guessing. The running time of contextual similarity, on the other hand, was over 10 minutes in our experiments because each window $w_{i}$ needs to be compared to over half a million documents in our corpus. The topical relevance and dependency feature approaches are versions of contextual similarity that use dimensionality reduction and so are much faster. Topical relevance required approximatley 0.5 seconds and the dependency feature model required about 1 second. Both running times are acceptable for this citation recommendation task considering the quality of the generated candidate citation contexts. The dependency feature model is the more expensive of the two because of the additional features it computes.

\subsection{Evaluating Recommended Citations}

One difficulty with evaluating how well candidate citation contexts cover the true citation locations is that there are many places where citations could have been (but were not) placed in a research paper (which is being used as the ground truth). This can happen when a similar idea had been previously referenced, when an idea is very common (e.g., few people cite Newton or Liebniz when the use derivatives), or when an author is unaware of related work. Thus in this section we evaluate the quality of candidate citation contexts differently from Section 5.3. Here we evaluate how well these candidate citation contexts can be used to recommend papers to cite. To generate recommendations from the candidate citation contexts, we use the relevance model [9] which ranks citations using Equation 1 from Section 3.

\subsubsection{Metrics}

Recall ( $\mathrm{R})$ : The average fraction of a testing document's bibliography that appears in top- $k$ recommended citations.

Relative co-cited probability $(\mathrm{P})$ : Some papers that are recommended may be relevant even if they are not cited by the testing document. This can occur because an author is unaware of related work or due to space constraints. Because of this, measures such as precision relative to the actual bibliography are not as meaningful. Thus in this paper, we will use a modified measure of accuracy based on the assumption that papers that are frequently co-cited are relevant to each other. Let $d$ be a testing document, $b_{i}$ a document cited by $d$, and $r_{j}$ a document that was recommended but not cited by $d$. We begin by computing the following quantity:

$p\left(r_{j} \mid b_{i}\right)=\frac{\text { number of papers in } D \text { citing both } b_{i} \text { and } r_{j}}{\text { number of papers in } D \text { citing } b_{i}}$.

For each testing document, we average $p\left(r_{j} \mid b_{i}\right)$ over all $<r_{j}, b_{i}>$ pairs and then we average over all testing documents. The result is the relative co-cited probability.

NDCG: The effectiveness of a recommendation system is also sensitive to the ordering of the relevant recommended citations (i.e., the most relevant citations should be highly ranked). This is not directly measured by the previous two metrics, and so we also use the normalized discounted cumulative gain (NDCG) to measure the ranking. The NDCG 
value of a ranked list at position $i$ is calculated as:

$$
N D C G @ i=Z_{i} \sum_{j=1}^{i} \frac{2^{r(j)}-1}{\log (1+j)},
$$

where $r(j)$ is the rating of the $j$-th document in the ranking list, and the normalization constant $Z_{i}$ is chosen so that the perfect list gets a NDCG score of 1 . Given a testing document $d$ and any other document $r$ from our corpus $D$, we use the average relative co-cited probability of $r$ with all original citations of $d$ to weigh the citation relevance score of $r$ to $d$. Then we sort the documents in $D$ with respect to this score (relevance to $d$ ) and assign a new score ( 5 point scale) to each document. If $R_{\max }$ is the maximum relevance of any $r \in D$ to $d$, then documents are assigned the scores $4,3,2,1,0$ if their relevance to $d$ falls within the ranges $\left(3 R_{\max } / 4, R_{\max }\right]$, $\left(R_{\max } / 2,3 R_{\max } / 4\right],\left(R_{\max } / 4, R_{\max } / 2\right],\left(0, R_{\max } / 4\right]$, and 0 , respectively. We report the average NDCG score over all testing documents.

\subsubsection{Result Analyses}

Figure 2 shows the citation recommendation performances of the baselines and methods for generating candidate citation contexts. Comparing with Table 3, we see that recommendation quality is correlated with the ability to cover the ground truth citation locations.

One exception is the baseline of guessing candidate citation contexts randomly. Its coverage precision/recall are not high but it is able to generate a decent bibliography anyway. Initially it may seem strange that randomly guessing the candidate citation contexts would result in a better recommended bibliography than the full-text baseline which treats the entire full-text of a testing paper as a candidate citation context. However, a testing document usually has multiple citation needs (e.g. references to statistical models and references to optimization algorithms for fitting the models) occurring in different places. Thus random guessing can result in two distinct citation contexts (one relevant to statistics and one relevant to optimization), each of which is relevant to a document in $D$, instead of one giant candidate citation context which is marginally related to many papers (as in the case of full-text).

It is also interesting to note that although the topical relevance method considerably outperforms the random method in finding citation locations, the bibliography that is subsequently generated is not much better than the method of randomly guessing candidate citation contexts. One plausible reason is that the topical relevance method (and to some extent the contextual similarity method and language models) are more susceptible to the redundancy mentioned in Section 4.4. That is, one theme of a paper highly relevant to other documents can recur many times, resulting in many similar (thus redundant) candidate citation contexts being chosen because of their high relevance score (meaning that citation contexts representing a different theme are likely to be left out). Random guessing on the other hand is not affected by such redundancy issues.

Additional evidence supporting this claim is that the dependency feature model (Section 4.4) produces a considerably better bibliography by incorporating long-range dependency features. Thus if a window is highly relevant to documents from the corpus $D$, but is also very similar to a previous window that was labeled as a candidate citation context, the new windows is less likely to also be (redundantly) labeled as a candidate citation context.

\section{CONCLUSIONS}

In this paper, we tackled the novel problem of automatically generating candidate citation contexts from the query manuscript to aid an academic literature search. Our approach used existing citation contexts as succinct descriptions of the contributions and ideas present in documents belonging to a large corpus. We are incorporating this approach into citation context identification and citation recommendation in CiteSeerX and we conducted extensive experiments to evaluate the performance of our approach.

\section{REFERENCES}

[1] S. Aya, C. Lagoze, and T. Joachims. Citation classification and its applications. In ICKM, 2005.

[2] C. Basu, H. Hirsh, W. Cohen, and C. Nevill-Manning. Technical paper recommendation: A study in combining multiple information sources. J. of Artificial Intelligence Research, 2001.

[3] D. Blei, A. Ng, and M. Jordan. Latent dirichlet allocation. J. Machine Learning Research, 2003.

[4] A. Broder, M. Fontoura, V. Josifovski, and L. Riedel. A semantic approach to contextual advertising. In SIGIR, 2007.

[5] K. Chandrasekaran, S. Gauch, P. Lakkaraju, and H. Luong. Concept-Based Document Recommendations for CiteSeer Authors. Adaptive Hypermedia and Adaptive Web-Based Systems, Springer, 2008.

[6] D. Cohn and T. Hofmann. The missing link - a probabilistic model of document content and hypertext connectivity. In NIPS, 2001.

[7] R. Durrett. Probability: Theory and Examples. Duxbury Press, 2nd edition, 1995.

[8] E. Erosheva, S. Fienberg, and J. Lafferty. Mixed membership models of scientific publications. PNAS, 2004.

[9] Q. He, J. Pei, D. Kifer, P. Mitra, and L. Giles. Context-aware citation recommendation. In $W W W$, 2010.

[10] S. Huang, G. Xue, B. Zhang, Z. Chen, Y. Yu, and W. Ma. Tssp: A reinforcement algorithm to find related papers. In WI, 2004.

[11] S. Kataria, P. Mitra, and S. Bhatia. Utilizing context in generative bayesian models for linked corpus. In AAAI, 2010.

[12] S. M. Katz. Estimation of probabilities from sparse data for the language model component of a speech recogniser. IEEE Transactions on Acoustics, Speech, and Signal Processing, 35(3):400-401, 1987.

[13] J. Kleinberg. Bursty and hierarchical structure in streams. In SIGKDD, 2002.

[14] D. Liben-Nowell and J. Kleinberg. The link prediction problem for social networks. In CIKM, 2003.

[15] S. McNee, I. Albert, D. Cosley, P. Gopalkrishnan, S. Lam, A. Rashid, J. Konstan, and J. Riedl. On the recommending of citations for research papers. In CSCW, 2002.

[16] R. Nallapati, A. Ahmed, E. Xing, and W. Cohen. Joint latent topic models for text and citations. In 


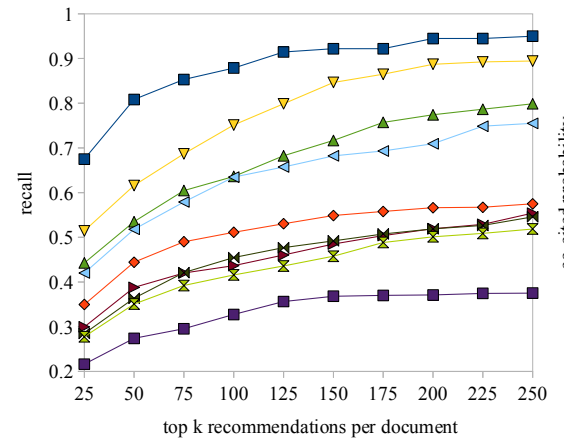

(a) recall

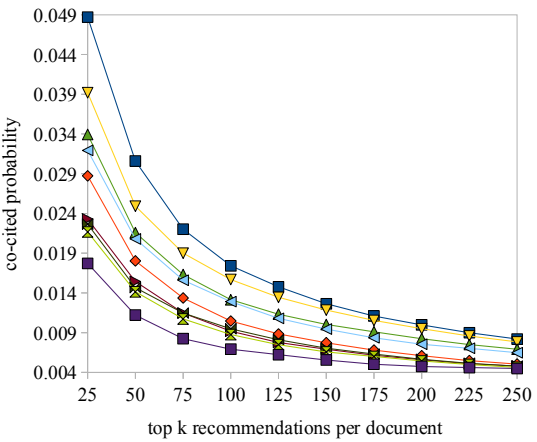

(b) co-cited probability



(c) NDCG

Figure 2: Compare performances for citation recommendation.

SIGKDD, 2008.

[17] J. M. Ponte and W. B. Croft. A language modeling approach to information retrieval. In Research and Development in Information Retrieval, 1998.

[18] J. R. Quinlan. C4.5: programs for machine learning. Morgan Kaufmann, 1993.

[19] B. Ribeiro-Neto, M. Cristo, P. B. Golgher, and E. S. de Moura. Impedance coupling in contenttargeted advertising. In SIGIR, 2005.

[20] C. Rijsbergen. The Geometry of Information Retrieval. Cambridge University Press, 2004.

[21] A. Ritchie. Citation context analysis for information retrieval. PhD thesis, University of Cambridge, 2008.

[22] B. Shaparenko and T. Joachims. Identifying the original contribution of a document via language modeling. In ECML, 2009.

[23] D. Simon. Optimal State Estimation: Kalman, H Infinity, and Nonlinear Approaches. Wiley-Interscience, 2006.

[24] T. Strohman, B. Croft, and D. Jensen. Recommending citations for academic papers. In SIGIR, 2007.

[25] J. Tang and J. Zhang. A discriminative approach to topic-based citation recommendations. In PAKDD, 2009.

[26] R. Torres, S. McNee, M. Abel, J. Konstan, and J. Riedl. Enhancing digitial libraries with techlens. In $J C D L, 2004$.

[27] V. von Brzeski, U. Irmak, and R. Kraft. Leveraging context in user-centric entity detection systems. In CIKM, 2007.

[28] F. Wang, B. Chen, and Z. Miao. A survey on reviewer assignment problem. In IEA/AIE, 2008.

[29] W. Yih, J. Goodman, and V. R. Carvalho. Finding advertising keywords on web pages. In $W W W, 2006$.

[30] Y. Zhao and G. Karypis. Hierarchical clustering algorithms for document datasets. Data Mining and Knowledge Discovery, 10(2):141-168, 2005.

[31] D. Zhou, S. Zhu, K. Yu, X. Song, B. Tseng, H. Zha, and L. Giles. Learning multiple graphs for document recommendations. In $W W W, 2008$.

\section{APPENDIX}

\section{A. PROOF OF LEMMA 4.1}

Suppose the $p_{i}$ are generated independently by the beta distribution $\operatorname{Beta}(\alpha, \beta)$ with $\alpha=\gamma p, \beta=\gamma(1-p)$. Beta $(\alpha, \beta)$ has mean $\frac{\alpha}{\alpha+\beta}=p$ and variance $\frac{\alpha \beta}{(\alpha+\beta)^{2}(\alpha+\beta+1)}=p(1-$ $p) /(\gamma+1)$. In Bayesian statistics, Beta $\left(\alpha+m_{i}, \beta+k_{i}-m_{i}\right)$ can be seen as the posterior distribution of the parameter $p_{i}$ of $\operatorname{Binomial}\left(p_{i}, k_{i}\right)$ after seeing $m_{i}$ successes in $k_{i}$ coin tosses. So, the expected value of $\operatorname{Binomial}\left(k_{i}, p_{i}\right)$ is $E\left(m_{i}\right)=p k_{i}$ and its variance is $\operatorname{Var}\left(m_{i}\right)=\operatorname{Var}\left(p_{i} k_{i}\right)+$ $E\left[k_{i} p_{i}\left(1-p_{i}\right)\right]$ (by the law of total variance [7]), which is given by

$$
\begin{aligned}
& \operatorname{Var}\left(p_{i} k_{i}\right)+E\left[k_{i} p_{i}\left(1-p_{i}\right)\right] \\
& =k_{i}^{2} \operatorname{Var}\left(p_{i}\right)+k_{i}\left(E\left[p_{i}\right]-E\left[p_{i}^{2}\right]\right) \\
& =k_{i}^{2} \operatorname{Var}\left(p_{i}\right)+k_{i}\left(E\left[p_{i}\right]-\frac{\alpha+1}{\alpha+\beta+1} E\left[p_{i}\right]\right) \\
& =\frac{k_{i}^{2} p(1-p)}{\gamma+1}+k_{i}\left(p-\frac{(\gamma p+1)}{\gamma+1} \cdot p\right) \\
& =\frac{k_{i}^{2} p(1-p)}{\gamma+1}+\frac{k_{i} p \gamma+k_{i} p-k_{i} p^{2} \gamma-k_{i} p}{\gamma+1} \\
& =\frac{k_{i}^{2} p(1-p)}{\gamma+1}+k_{i} p(1-p) \frac{\gamma}{\gamma+1} \\
& =\frac{k_{i} p(1-p)\left(k_{i}+\gamma\right)}{\gamma+1}
\end{aligned}
$$

Thus $E\left[m_{i} / k_{i}\right]=p$ and

$$
\operatorname{Var}\left(\frac{m_{i}}{k_{i}}\right)=\frac{1}{k_{i}^{2}} \operatorname{Var}\left(m_{i}\right)=\frac{p(1-p)\left(k_{i}+\gamma\right)}{k_{i}(\gamma+1)} .
$$

It is easy to see that the covariance of $m_{i} / k_{i}$ and $m_{j} / k_{j}$ is 0 for $i \neq j$. Now, we are looking for the convex combination $a_{1}\left(m_{1} / k_{1}\right)+\cdots+a_{n}\left(m_{n} / k_{n}\right)$ with minimum variance. This is a simple optimization problem with lagrange multipliers and the solution is that $a_{i} \propto 1 / \operatorname{Var}\left(m_{i} / k_{i}\right)$ [23]. This gives

$$
\begin{aligned}
\hat{p} & =\sum_{i=1}^{n} \frac{\frac{1}{\operatorname{Var}\left(m_{i} / k_{i}\right)}}{\sum_{j=1}^{n} \frac{1}{\operatorname{Var}\left(m_{j} / k_{j}\right)}} \cdot \frac{m_{i}}{k_{i}} \\
& =\frac{1}{\sum_{j=1}^{n} \frac{k_{j}(\gamma+1)}{p(1-p)\left(k_{j}+\gamma\right)}} \sum_{i=1}^{n} \frac{m_{i}(\gamma+1)}{p(1-p)\left(k_{i}+\gamma\right)} \\
& =\frac{1}{\sum_{j=1}^{n} \frac{k_{j}}{k_{j}+\gamma}} \sum_{i=1}^{n} \frac{m_{i}}{k_{i}+\gamma} .
\end{aligned}
$$

